\title{
RIGIDITY FOR ODD-DIMENSIONAL SOULS
}

\author{
KRISTOPHER TAPP
}

\begin{abstract}
We prove a new rigidity result for an open manifold $M$ with nonnegative sectional curvature whose soul $\Sigma \subset M$ is odd-dimensional. Specifically, there exists a geodesic in $\Sigma$ and a parallel vertical plane field along it with constant vertical curvature and vanishing normal curvature. Under the added assumption that the Sharafutdinov fibers are rotationally symmetric, this implies that for small $r$, the distance sphere $B_{r}(\Sigma)=\{p \in M \mid \operatorname{dist}(p, \Sigma)=r\}$ contains an immersed flat cylinder, and thus could not have positive curvature.
\end{abstract}

\section{INTRODUCTION}

In this paper, we prove the following rigidity result for odd-dimensional souls:

Theorem 1.1. If $M$ is an open manifold with nonnegative sectional curvature whose soul $\Sigma \subset M$ is odd-dimensional, then there exists a geodesic $\gamma(t)$ in $\Sigma$, and orthonormal parallel vertical vector fields $V(t), W(t)$ along $\gamma(t)$ ("vertical" means orthogonal to $T_{\gamma(t)} \Sigma$ for each $\left.t\right)$ such that $\langle R(V(t), W(t)) W(t), V(t)\rangle$ is constant and $R(V(t), W(t)) \gamma^{\prime}(t)=0$ for all $t$, where $R$ denotes the curvature tensor of $M$.

We will discuss why this rigidity provides infinitesimal evidence of an affirmative answer to:

Question 1.2. For each small $r>0$, must the image, $C_{r}$, of the immersed cylinder

$$
\sigma(t, \theta)=\left\{\exp _{\gamma(t)}((r \cos \theta) V(t)+(r \sin \theta) W(t)) \mid t \in \mathbb{R}, \theta \in S^{1}\right\}
$$

be "flat" in the sense that the Jacobi field $\frac{\partial \sigma}{\partial \theta}$ along each of the $\{\theta=$ constant $\}$ geodesics out of which $C_{r}$ is ruled is a parallel Jacobi field in $M$ ?

Notice that $C_{r}$ is not totally geodesic in $M$, but might be totally geodesic in the distance sphere $B_{r}(\Sigma)=\{p \in M \mid \operatorname{dist}(p, \Sigma)=r\}$. Recall that $B_{r}(\Sigma)$ is convex in $M$ by 2 and thus always inherits nonnegative curvature. An affirmative answer to Question 1.2 means that $B_{r}(\Sigma)$ could not have strictly positive curvature when $\Sigma$ is odd-dimensional. However, examples are known for which $B_{r}(\Sigma)$ has some points of positive curvature. Specifically, Wilking constructed in [6] a metric with almost positive curvature on $S^{2} \times S^{3}$ that can be extended to a nonnegatively curved metric on $S^{3} \times \mathbb{R}^{3}$ (see [5] for an alternative description of his metric that makes the extendability more obvious).

We provide an affirmative answer to Question 1.2 under the added hypothesis that the intrisic metric on each Sharafutdinov fiber is rotationally symmetric (which means $O(k)$-invariant, where $k$ is the dimension of the fiber). We do not need to assume that the fibers all have the same rotationally symmetric metric.

Date: November 26, 2018.

1991 Mathematics Subject Classification. Primary 53C20.

Key words and phrases. nonnegative curvature, soul, flats. 
Theorem 1.3. With the assumptions and terminology of Theorem [1.1 and Question 1.2. if the Sharafutdinov fibers are all rotationally symmetric, then for each $r>0, C_{r}$ is flat; further, the fibers along $\gamma$ are mutually isometric.

We are pleased to thank Ilya Bogdanov for the proof of Lemma 3.1 We would also like to thank Igor Belegradek and Luis Guijarro for helpful discussions about this work.

\section{BACKGROUND}

For the remainder of this paper, $M$ will denote an open manifold with nonnegative curvature. According to [1, $M$ is diffeomorphic to the total space of the normal bundle of its soul, $\Sigma \subset M$. We will denote this normal bundle as $\nu(\Sigma)$, and its fiber at $p \in \Sigma$ as $\nu_{p}(\Sigma)=\left\{V \in T_{p} M \mid V \perp T_{p} \Sigma\right\}$. Our main tool is the following "soul inequality" for the curvature tensor, $R$, of $M$, found in [4]:

Proposition 2.1 (4]). For all $p \in \Sigma, X, Y \in T_{p} \Sigma$ and $V, W \in \nu_{p}(\Sigma)$, we have:

$\left(D_{X} R\right)(X, Y, W, V)^{2} \leq\left(|R(W, V, X)|^{2}+\frac{2}{3}\left(D_{X} D_{X} R\right)(W, V, V, W)\right) \cdot R(X, Y, Y, X)$.

Here, we are considering $R$ sometimes as a function from $\left(T_{p} M\right)^{3} \rightarrow T_{p} M$ and sometimes from $\left(T_{p} M\right)^{4} \rightarrow \mathbb{R}$, in the obvious way. This inequality was originally expressed in [4] in a manner which more explicitly distinguished the three different types of curvature that it relates:

$$
\left\langle\left(D_{X} R^{\nabla}\right)(X, Y) W, V\right\rangle^{2} \leq\left(\left|R^{\nabla}(W, V) X\right|^{2}+\frac{2}{3}\left(D_{X} D_{X} k^{f}\right)(W, V)\right) \cdot k_{\Sigma}(X, Y) .
$$

Here, $k_{\Sigma}$ and $k^{f}$ denote respectively the unnormalized intrinsic sectional curvature of $\Sigma$ and of the Sharafutinov fiber, $\exp \left(\nu_{p}(\Sigma)\right)$. Notice that the intrinsic equals the extrinsic curvature because $\Sigma$ is totally geodesic, and because each Sharafutinov fiber is totally geodesic a point of the soul. To interpret the $k_{f}$ term, just extend $W, V$ to parallel fields $W(t), V(t)$ along the geodesic in $\Sigma$ in the direction of $X$, and notice that $\left(D_{X} D_{X} k^{f}\right)(W, V)$ equals the second derivative at $t=0$ of the vertical curvature function $t \mapsto k^{f}(W(t), V(t))$.

Further, $R^{\nabla}: T_{p} \Sigma \times T_{p} \Sigma \times \nu_{p}(\Sigma) \rightarrow \nu_{p}(\Sigma)$ denotes the "normal curvature tensor" which means the curvature tensor of the induced connection, $\nabla$, in $\nu(\Sigma)$, so that $R^{\nabla}(W, V) X \in T_{p} \Sigma$ can be defined as the unique vector such that $\left\langle R^{\nabla}(W, V) X, Y\right\rangle=$ $\left\langle R^{\nabla}(X, Y) W, V\right\rangle$ for all $Y \in T_{p} \Sigma$. The fact that $R^{\nabla}$ is just a restriction of $R$ follows from the fact that $\Sigma$ is totally geodesic.

\section{Proof of Theorem 1.1}

We will require the following fact about smooth functions:

Lemma 3.1. Suppose $f, g: \mathbb{R} \rightarrow \mathbb{R}$ are smooth functions. Assume that $f(0)=0$ and that $g(t)$ has a global maximum at $t=0$. Assume for all $t \in \mathbb{R}$ that:

$$
f^{\prime}(t)^{2} \leq f(t)^{2}+g^{\prime \prime}(t) .
$$

Then $f$ and $g$ are both constant functions.

The following proof is due to Ilya Bogdanov, communicated via mathoverflow.net. Notice that the Lemma is very simple to prove for analytic functions. 
Proof. Assume without loss of generality that $g(0)=0$. Using Cauchy-Schwarz, we have that for all $t \in(0,1)$ :

$$
\int_{0}^{t}\left(f(s)^{2}+g^{\prime \prime}(s)\right) \mathrm{d} s \geq \int_{0}^{t} f^{\prime}(s)^{2} \mathrm{ds} \geq \frac{\left(\int_{0}^{t} f^{\prime}(s) \mathrm{ds}\right)^{2}}{\int_{0}^{t} 1^{2} \mathrm{ds}}=\frac{f(t)^{2}}{t} \geq f(t)^{2} .
$$

Therefore,

$$
g^{\prime}(t) \geq f(t)^{2}-\int_{0}^{t} f(s)^{2} \mathrm{ds}
$$

Define $h(t)=\int_{0}^{t} f(s)^{2} \mathrm{ds}$, so the previous equation becomes:

$$
g^{\prime}(t) \geq h^{\prime}(t)-h(t)
$$

Since $h$ is monotonic, integrating the previous equation equation gives:

$$
g(t) \geq h(t)-\int_{0}^{t} h(s) \mathrm{ds} \geq h(t)-t h(t) \geq 0 .
$$

Since $g(0)=0$ is a global maximum, this implies that $g(t)=h(t)=f(t)=0$ for all $t \in(0,1)$, and thus clearly also for all $t \in \mathbb{R}$.

We are now prepared to prove our first theorem.

Proof of Theorem 1.1. Chose $p \in \Sigma$ and orthogonal unit-length vectors $W, V$ in $\nu_{p}(\Sigma)$ such that $k^{f}(W, V)$ is maximal (among all such $p, V, W$ ), which implies that $\left(D_{X} D_{X} k^{f}\right)(W, V)=0$ for all $X \in T_{p} \Sigma$. Since $X \mapsto R^{\nabla}(W, V) X$ is a skewsymmetric endomorphism of the odd-dimensional vector space $T_{p} \Sigma$, there exists a unit-length vector $X \in T_{p} \Sigma$ such that $R(W, V) X=0$. For any $Y \in T_{p} \Sigma$, the right side of the soul inequality vanishes for the vectors $\{X, Y, W, V\}$, and therefore the left side also vanishes.

Let $\gamma(t)$ denote the geodesic in $\Sigma$ with $\gamma(0)=p$ and $\gamma^{\prime}(0)=X$. Let $X(t)=\gamma^{\prime}(t)$, which is the parallel transport of $X$ along $\gamma(t)$. Let $W(t), V(t)$ denote the parallel transports of $W, V$ along $\gamma(t)$. Define:

$$
g(t)=\frac{2}{3} \cdot k^{f}(V(t), W(t)) \text { and } f(t)=\left|R^{\nabla}(W(t), V(t)) X(t)\right| .
$$

Let $C$ denote the maximum sectional curvature of $\Sigma$. For any unit-length $Y(t) \in$ $T_{\gamma(t)} \Sigma$, the soul inequality gives:

$$
\left\langle\frac{D}{d t}\left(R^{\nabla}(W(t), V(t)) X(t)\right), Y(t)\right\rangle^{2} \leq\left(f(t)^{2}+g^{\prime \prime}(t)\right) \cdot C .
$$

In particular, choosing $Y$ parallel to $\frac{D}{d t}\left(R^{\nabla}(W(t), V(t)) X(t)\right)$ gives:

$$
\left(f^{\prime}(t)\right)^{2} \leq\left(f(t)^{2}+g^{\prime \prime}(t)\right) \cdot C .
$$

Lemma 3.1 now implies that $f$ is identically zero and that $g$ is constant. 


\section{Proof of Theorem 1.3}

In this section, we prove our second theorem.

Proof of Theorem 1.3. Let $\gamma(t), X(t), V(t)$ and $W(t)$ be as in the proof of Theorem 1.1. Choose a fixed $r>0$. Let $\exp ^{\perp}: \nu(\Sigma) \rightarrow M$ denote the normal exponential map. Let $A$ and $T$ denote the fundamental tensors of the Sharafutdinov map $\pi: M \rightarrow \Sigma$.

Let $\bar{\gamma}(t)=\exp ^{\perp}(r \cdot W(t))$, which is one of the $\pi$-horizontal geodesics out of which the cylinder $C_{r}$ is ruled. In fact, since $\{W, V\}$ can be replaced with any orthonormal basis of their span, we can consider $\bar{\gamma}(t)$ as an arbitrary one of the geodesics which rule $C_{r}$.

Let $\bar{X}(t)=\bar{\gamma}^{\prime}(t)$ and let $\bar{V}(t)=\left(\exp _{*}^{\perp}\right)(V(t))$, where $\left(\exp _{*}^{\perp}\right)$ denotes the derivative of $\exp ^{\perp}$ at the relevant point, which in this case is $r \cdot W(t)$. Notice that $\bar{V}(t)$ is a $\pi$-vertical vector field along $\bar{\gamma}(t)$. In fact, $\bar{V}(t)$ is a vertical Jacobi field along $\bar{\gamma}(t)$ because it is the variational field of the family of horizontal geodesics by which the cylinder $C_{r}$ is ruled. The covariant derivative of $\bar{V}(t)$ along $\bar{\gamma}(t)$ has horizontal and vertical components determined respectively by the $A$ and $T$-tensors of $\pi$ :

$$
\bar{V}^{\prime}(t)=A_{\bar{X}(t)} \bar{V}(t)+T_{\bar{V}(t)} \bar{X}(t) .
$$

To prove that $C_{r}$ is flat (in the sense of Question 1.2) it will suffice to show that $\bar{V}^{\prime}(t)=0$ for all $t$. In fact, it will suffice to prove this just for $t=0$.

We will sometimes write $X, V, W, \bar{X}$ and $\bar{V}$ for the values of these fields at $t=0$. By the Jacobi Equation, the sectional curvature of the vertizontal plane spanned by $\bar{X}$ and $\bar{V}$ equals:

$$
k(\bar{X}, \bar{V})=-\left\langle\bar{V}^{\prime \prime}, \bar{V}\right\rangle .
$$

We first claim that $A_{\bar{X}} \bar{V}=0$. Recall that for any horizontal vectors $\bar{Z}_{1}, \bar{Z}_{2}$ at $\bar{\gamma}(0)$, Proposition 1.7 of [3] implies that the $A$-tensor can be described as:

$$
A_{\bar{Z}_{1}} \bar{Z}_{2}=\frac{1}{2}\left(\exp _{*}^{\perp}\right)\left(R^{\nabla}\left(\pi_{*} \bar{Z}_{1}, \pi_{*} \bar{Z}_{2}\right) W\right) .
$$

For any horizontal vector $\bar{Y}$ at $\bar{\gamma}(0)$, let $Y=\pi_{*} \bar{Y}$ and notice that:

$$
\left\langle A_{\bar{X}} \bar{V}, \bar{Y}\right\rangle=-\left\langle A_{\bar{X}} \bar{Y}, \bar{V}\right\rangle=-\frac{1}{2}\left\langle\left(\exp _{*}^{\perp}\right)\left(R^{\nabla}(X, Y) W\right),\left(\exp _{*}^{\perp}\right) V\right\rangle .
$$

This equals zero because

$$
\left\langle R^{\nabla}(X, Y) W, V\right\rangle=\left\langle R^{\nabla}(W, V) X, Y\right\rangle=0
$$

and because $\exp _{*}^{\perp}$ preserves orthogonality due to our assumption that the fibers are rotationally symmetric (and thus that the distance spheres in these fibers are round).

Since the $A$-tensor term vanishes, Equation 4.1 becomes $\bar{V}^{\prime}(t)=T_{\bar{V}(t)} \bar{X}(t)$, and it remains to prove that this $T$-tensor term vanishes. Let $\bar{U}$ be an arbitrary vertical vector at $\bar{\gamma}(0)$ which is perpendicular to $\bar{V}$ and to the radial direction. Let $\bar{U}(t)$ denote the extension of $\bar{U}(0)=\bar{U}$ to the "hononomy Jacobi field" along $\bar{\gamma}(t)$; that is, the variational vector field of the family of lifts of $\gamma(t)$ to horizontal geodesics beginning at points along a curve in the Sharafutdinov fiber tangent to $\bar{U}$. Notice that for each $t_{0}, \bar{V}\left(t_{0}\right)$ and $\bar{U}\left(t_{0}\right)$ are the images of $\bar{V}$ and $\bar{U}$ under the derivative at $\bar{\gamma}(0)$ of the "holonomy diffeomorphism" $h_{\gamma}: \pi^{-1}(\gamma(0)) \rightarrow \pi^{-1}\left(\gamma\left(t_{0}\right)\right)$. Since 
the fibers are rotationally symmetric, $h_{\gamma}$ (restricted to the spheres of radius $r$ ) is simply a rescaling map from a round spheres to a round sphere, and thus preserve orthogonality. Therefore,

$$
\left\langle\bar{U}\left(t_{0}\right), \bar{V}\left(t_{0}\right)\right\rangle=\left\langle d\left(h_{\gamma}\right)_{\bar{\gamma}(0)}(\bar{U}), d\left(h_{\gamma}\right)_{\bar{\gamma}(0)}(\bar{V})\right\rangle=\langle\bar{U}, \bar{V}\rangle=0 .
$$

It follows that

$$
\left\langle\bar{V}^{\prime}(t), \bar{U}(t)\right\rangle=\left\langle T_{\bar{V}(t)} \bar{X}(t), \bar{U}(t)\right\rangle=(1 / 2) \frac{d}{d t}\langle\bar{V}(t), \bar{U}(t)\rangle=0 .
$$

This means that $\bar{V}^{\prime}(t)$ is parallel to $\bar{V}(t)$, so we can write $\bar{V}(t)=s(t) P(t)$ for some positive-valued function $s(t)$ and some parallel vector field $P(t)$. Equation 4.2 implies that $s^{\prime \prime}(t) \leq 0$ for all $t$. Since $s(t)$ is nowhere zero, we conclude that $s(t)$ is constant. Thus, $\bar{V}(t)$ is a parallel Jacobi field.

It remains to explain why the Sharafutdinov fibers along $\gamma$ are mutually isometric. Since $|\bar{V}(t)|$ is constant, the $\{t=$ constant $\}$ circles in the cylinder $C_{r}$ must all have the same length. But these are great circle in the round spheres $\exp ^{\perp}\left(\nu_{\gamma(t)}(\Sigma)\right) \cap B_{r}(\Sigma)$, so these round spheres must all have the same diameters. Since this is true for each $r$, the Sharafutdinov fibers along $\gamma$ have isometric distance spheres at all distances, so the fibers are mutually isometric.

\section{REFERENCES}

1. J. Cheeger and D. Gromoll, On the structure of complete manifolds of nonnegative curvature, Ann. of Math. 96 (1972), 413-443.

2. L. Guijarro and G. Walschap, The metric projection onto the soul, Tans. Amer. Math. Soc. 352 (2000), no. 1, 55-69.

3. M. Strake and G. Walschap, $\Sigma$-flat manifolds and Riemannian submersions, Manuscripta Math. 61 315-325.

4. K. Tapp, Conditions of nonnegative curvature on vector bundles and sphere bundles, Duke Math. Journal. 116, no. 1 (2003), 77-101.

5. K. Tapp, Quasi-positive curvature on homogeneous bundles, J. Diff. Geom, 65 (2003), pp. 273-287.

6. B. Wilking, Manifolds with positive sectional curvature almost everywhere, Invent. Math. 148 (2002), 117-141.

Department of Mathematics, Saint Joseph's University, 5600 City Avenue PhiladelPHIA, PA 19131

E-mail address: ktapp@sju.edu 